\title{
Study of Graded Algebras and General Linear Group with Lie Superalgebras and R-Algebra
}

\author{
Khondokar M. Ahmed ${ }^{1 *}$, S. K. Rasel ${ }^{2}$, Jyoti Das ${ }^{3}$, Saraban Tahura ${ }^{4}$ and Salma Nasrin ${ }^{5}$ \\ ${ }^{1,5}$ Department of Mathematics, University of Dhaka, Dhaka-1000, Bangladesh. \\ ${ }^{2}$ Department of General Educational Development, Daffodil International University, Dhaka-1207, Bangladesh. \\ ${ }^{3}$ Department of Mathematics, Comilla University, Comilla, Bangladesh. \\ ${ }^{4}$ Department of Natural Sciences, University of Information Technology and Sciences, Dhaka 1212, Bangladesh.
}

(Received : 22 July 2018 ; Accepted : 7 January 2020 )

\begin{abstract}
Some elements of theory of $\mathbb{Z}_{2}$-graded rings, modules and algebras. $\mathbb{Z}_{2}$-graded tensor algebra, Lie superalgrbras and matrices with entries in a $\mathbb{Z}_{2}$-graded commutative ring are treated in our present paper. At last a Theorem 4.4.on the set of square matrices in the graded $R$-algebra $M_{R}[m \mid n]$ is established.
\end{abstract}

Keywords: $\mathbb{Z}_{2}$-graded rings, modules, commutative ring and graded algebras, tensor calculus, general graded linear group $G L[m \mid n]$, the set of graded matrices $M_{R}[(p+q) \times(m+n)]$ and graded $R$-algebra.

\section{Introduction}

Nowadays a large body of literature is available concerning graded algebras, mainly over the real or complex numbers (usually called superalgebras), their representations, etc. Classical references are [3], [6], [7], [8], [10]. The most common notations and basic results are treated in this article.

\section{Graded Algebraic Structures}

In general, given an arbitrary group $G$, we can introduce $G$ graded algebraic objects [5], [10]. Since in order to develop a 'supergeometry' only $\mathbb{Z}_{2}$-graded structures are needed, we shall only consider here that particular case. We shall assume as a rule that

$$
\text { graded } \equiv \mathbb{Z}_{2}-\text { graded }
$$

Definition 2.1. A ring $(R,+, \cdot)$ is said to be graded if $(R,+)$ has two subgroups $R_{0}$ and $R_{1}$ such that $R=R_{0} \oplus$ $R_{1}$ and $R_{\alpha}, R_{\beta} \subset R_{\alpha+\beta}$ for all $\alpha, \beta \in \mathbb{Z}_{2}$.

An element $a \in R$ is said to be homogeneous if either $a \in R_{0}$ or $a \in R_{1}$. On the set $h(R)$ of homogeneous elements an application || is defined by

$$
\begin{gathered}
||: h(R) \rightarrow \mathbb{Z}_{2} \\
a \mapsto \alpha \Leftrightarrow a \in R_{\alpha} .
\end{gathered}
$$

The elements of degree 0 and 1 are called even and odd respectively.

Obviously, any ring $R$ can be trivially graded: $R_{0}=R$, $R_{1}=\{0\}$.

Example 2.2. Let $R$ be a $\mathbb{Z}$-graded ring, namely, $R=$ $\bigoplus_{p \in \mathbb{Z}} \hat{R}_{p}$ and $\hat{R}_{p} . \hat{R}_{q} \subset \hat{R}_{p+q}$ then $R$ can be graded by takig $R_{0}$ as the sum of the even components and $R_{1}$ as the sum of the odd ones.

For any graded ring $R$, a graded commutator $<,>: R \times R \rightarrow$ $R$ is defined by letting

$$
<a, b>=a b-(-1)^{|a||b|} b a \forall a, b \in h(R)
$$

The centre of $R$ is defined as the set

$$
C(R) \equiv\{a \in R|<a, b\rangle=0 \forall b \in R\},
$$

i.e. $C(R)$ is the set of the elements of $R$ which graded commute with any other elements.

A graded ring $R$ is said to be graded-commutative if $\langle a, b\rangle=0 \forall a, b \in R$, that is, if $C(R)=R$.

Let $R$ be a graded ring and $M$ be a left(right) $R$-module.

Definition 2.3. Mis a left (right) graded $R$-module if it has two subgroups $M_{0}$ and $M_{1}$ such that $M=M_{0} \oplus M_{1}$ and for all $\alpha, \beta \in \mathbb{Z}_{2}$, one has $R_{\alpha} M_{\beta} \subset M_{\alpha+\beta}\left(M_{\alpha} R_{\beta} \subset M_{\alpha+\beta}\right)$.

If $R$ is graded-commutative, which we shall henceforth assume, we shall use the term 'graded $R$-module' without ambiguity.

Having fixed two graded $R$-modules $M$ and $N$, we say that a morphism $f: M \rightarrow N$ is $R$-linear on the right if $f(x a)=$ $f(x) a$ for all $x \in M$ and $a \in R$. Unless otherwise stated, by 'linear' we mean 'linear on the right'. Moreover, we say that $f$ has degree $|f|=\beta \in \mathbb{Z}_{2}$, if $f\left(M_{\alpha}\right) \subset N_{\alpha+\beta}$ for all $\alpha \in \mathbb{Z}_{2}$. The set $\operatorname{Hom}(M, N)$ of $R$-linear morphisms $M \rightarrow$ $N($ that will be denoted simply by $\operatorname{Hom}(M, N)$ ) has a natural grading, with $f \in \operatorname{Hom}(M, N)_{\alpha}$ whenever $|f|=\alpha$. If $R$ is graded-commutative, $\operatorname{Hom}(M, N)$ is a graded $R$-module, with the multiplication rule $(a f)(x)=a f(x)$.

One of the most basic results in commutative ring theory, namely the Nakayama lemma, can be generalized to the graded setting. Let us define the radical of a gradedcommutative ring $R$ as the graded ideal $\mathcal{R}$ obtained by intersecting all maximal graded ideals of $R$.

Proposition 2.4.(Graded Nakayama Lemma) Let $R$ be $a$ graded-commutative ring $R, I$ be a graded ideal contained in the radical $\mathcal{R}$ of $R$ and $M$ be a graded finitely generated $R$-module. 
(a) If IM $=M$, then $M=0$.

(b) If $N$ is a graded submodule of Mand $M=I M+N$, then $M=N$.

(c) If $x^{1}, \ldots, x^{m}$ are even elements and $y^{1}, \ldots, y^{n}$ are odd elements in $M$ such that the images $\left(\bar{x}^{1}, \ldots, \bar{x}^{m}, \bar{y}^{1}, \ldots, \bar{y}^{n}\right)$ are generators of $M /$ IMover $R / I$, then $\left(x^{1}, \ldots, x^{m}, y^{1}, \ldots, y^{n}\right)$ are generators of Mover $R$.

Definition 2.5. A graded Rmodule $F$ is said to be free if it has a basis formed by homogeneous elements.

A basis of $F$ of finite cardinality is of type $(m, n)$, if it is formed by $m$ even elements $\left\{f_{i}^{0} \in F_{0} \mid i=1, \ldots, m\right\}$ and $n$ odd elements $\left\{f_{\alpha}^{1} \in F_{1} \mid \alpha=1, \ldots, n\right\}$.

We have a canonical isomorphism

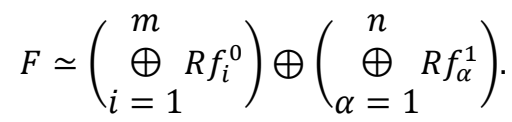

For each pair of natural numbers $m, n$ such that $m+n=p$, the $R$-module $R^{p}$ can be regarded as a free graded $R$ module endowed with a basis of type $(m, n)$, by letting,

$$
\begin{gathered}
\left(R^{m+n}\right)_{0} \equiv R^{m, n}=R_{0}^{m} \oplus R_{1}^{n} ; \\
\left(R^{m+n}\right)_{1} \equiv R^{\bar{m}, \bar{n}}=R_{0}^{n} \oplus R_{1}^{m}
\end{gathered}
$$

$R^{m+n}$ equipped with this gradation will be denoted by $R^{m \mid n}$.

Example 2.6. (cf. [5]) Let $R$ be a commutative ring, and $M$ be an $R$-module. The exterior algebra of $M$ over $R$, denoted by $\wedge_{R} M$, is a $\mathbb{Z}$-graded algebra, namely $\bigoplus_{p \in \mathbb{Z}} \wedge_{R}^{p} M$, and is alternating, i.e. $x^{2}=0$ for all $x \in \wedge_{R}^{2 p+1} M$. If $M$ is free and finitely generated, with a basis $\left\{e_{i} \mid i=1, \ldots, N\right\}$, then $\wedge_{R} M$ is a free finitely generated $R$-module, with a canonical basis(relative to the basis $\left\{e_{i}\right\}$ ) which can be described as follows. Let $\Xi_{\mathrm{N}}$ denote the set

$$
\left\{\begin{array}{c}
\mu:\{1, \ldots, r\} \rightarrow \\
\{1, \ldots, N\} \text { strictly increasing }
\end{array} \mid 1 \leq r \leq N\right\} \cup\left\{\mu_{0}\right\},
$$

where $\mu_{0}$ is the empty sequence, and let

$$
\beta_{\mu}=e_{\mu(1)} \wedge \ldots \wedge e_{\mu(r)} \text { for } \mu \neq \mu_{0}, \beta_{\mu_{0}}=1 \text {. }
$$

Then $\left\{\beta_{\mu} \mid \mu \in \Xi_{N}\right\}$ is the canonical basis of $\Lambda_{R} M$.

The cases $R=\mathbb{R}$ and $R=\mathbb{C}$ have a particular interest and deserve ad hoc notations:

$\wedge_{\mathbb{R}} \mathbb{R}^{L} \equiv B_{L} ; \wedge_{\mathbb{C}} \mathbb{C}^{L} \equiv C_{L}$

$B_{L}$ is a vector space, with a canonical basis obtained from the canonical basis of $\mathbb{R}^{L}$ according to the above described procedure. If $\mathbb{m}_{L}$ is the ideal of nilpotentsof $B_{L}$, the vector space direct sum decomposition $B_{L}=\mathbb{R} \bigoplus \mathbb{m}_{L}$ defines two projections

$\sigma: B_{L} \rightarrow \mathbb{R} ; \quad s: B_{L} \rightarrow \mathbb{R}_{L}$

which are sometimes called body and soul maps.

Tensor Products: Let us recall that we are considering a graded-commutative ring $R$. The graded tensor product of two graded $R$-modules $M, N$ is by definition the usual tensor product $M \bigoplus_{R} N$, obtained by regarding $M$ as a right module, and $N$ as a left module, equipped with the gradation

$$
\left(M \oplus_{R} N\right)_{\gamma}=\bigoplus_{\alpha+\beta=\gamma}\left\{\sum m_{i} \otimes n_{j} \mid m_{i} \in M_{\alpha}, n_{j} \in N_{\beta}\right\}
$$

Evidently, $M \otimes_{R} N$ has a natural structure of graded $R$ module:

$$
\begin{aligned}
& \begin{aligned}
& a(x \otimes y)=a x \otimes y=(-1)^{|a||x|} x a \otimes y \\
&=(-1)^{|a||x|} x \otimes a y \\
&=(-1)^{|a|(|x|+|y|)}(x \otimes y) a .
\end{aligned}
\end{aligned}
$$

The graded tensor product can be characterized as a 'universal object'. To this end, given graded $R$-modules $M, N$ and $Q$, we introduce the set $\mathcal{L}(M, N ; Q)_{\alpha}$ (with $\alpha \in$ $\mathbb{Z}_{2}$ ) of the graded $R$-bilinear morphisms $f: M \times N \rightarrow Q$, homogeneous of degree $\alpha$ : if $f \in \mathcal{L}(M, N ; Q)_{\alpha}$, then $f$ is a morphism of degree $\alpha$ such that $f(x a, y)=f(x, a y)=$ $(-1)^{|a||y|} f(x, y) a$ for all $a \in R$. The set

$$
\mathcal{L}(M, N ; Q) \equiv \mathcal{L}(M, N ; Q)_{0} \oplus \mathcal{L}(M, N ; Q)_{1}
$$

is endowed with a structure of graded $R$-module by enforcing the multiplication rule $(f a)(x, y)=f(a x, y)$. In the same way, if $M_{1}, \ldots, M_{n}, Q$ are graded $R$-modules, we define the graded $R$-module $\mathcal{L}\left(M_{1}, \ldots, M_{n} ; Q\right)$ formed by the graded $R$-multilinear morphisms $M_{1} \times \cdots \times M_{n} \rightarrow Q$.

Proposition 2.7. There are natural isomorphisms in the category $R-G$ Module

$$
\begin{aligned}
\mathcal{L}(M, N ; Q) \simeq \operatorname{Hom}_{R}\left(M \otimes_{R} N, Q\right) \\
\\
\simeq \operatorname{Hom}_{R}\left(M, \operatorname{Hom}_{R}(N, Q)\right) .
\end{aligned}
$$

Proposition 2.8. Let $M, M^{\prime}, M^{\prime \prime}$ be graded $R$-modules; the following natural isomorphisms of graded $R$-modules hold:

(a) $\quad M \otimes_{R} M^{\prime} \simeq M^{\prime} \bigotimes_{R} M$, achieved by the morphism

(b) $\quad\left(M \otimes_{R} M^{\prime}\right) \bigotimes_{R} M^{\prime \prime}$

$$
x \otimes x^{\prime} \mapsto(-1)^{|x| \mid x^{\prime}} x_{x^{\prime}} \otimes x ;
$$

$\simeq M^{\prime} \bigotimes_{R}\left(M^{\prime} \bigotimes_{R} M^{\prime \prime}\right)$, achieved by the morphism $\left(x \otimes x^{\prime}\right) \otimes x^{\prime \prime} \mapsto x \otimes\left(x^{\prime} \otimes x^{\prime \prime}\right) ;$

(c) $\quad R \otimes_{R} M \simeq M \simeq M \otimes_{R} R$.

If $f: M \rightarrow P, g: N \rightarrow Q$ are morphisms of graded modules over a graded ring $R$, the tensor product $f \otimes g: M \otimes_{R} N \rightarrow$ $P \otimes_{R} Q$ is the morphism defined by the condition

$(f \otimes g)(m \otimes n)=(-1)^{|g||m|} f(m) \otimes g(n)$.

\section{Graded Algebras and Graded Tenso Calculus}

Let $R$ be a graded-commutative ring.

Definition 3.1. A graded R-algebra Pis a graded $R$-module endowed with a graded $R$-bilinear multiplication

$$
\begin{gathered}
P \otimes P \rightarrow P \\
x \otimes y \mapsto x \cdot y .
\end{gathered}
$$


A graded $R$-algebra $P$ is said to be graded-commutative if all graded commutators

$$
<x, y>=x \cdot y-(-1)^{|x||y|} y \cdot x,
$$

defined on the analogy of equation (2.1), vanish.

Example 3.2. The graded module $B_{L}\left(C_{L}\right)$ in traduced in Example 2.6. , equipped with the exterior product, is a graded-commutative $\mathbb{R}$-algebra( $\mathbb{C}$-algebra).

The graded tensor product $P \otimes_{R} Q$ of two graded $R$ algebras $P$ and $Q$ is defined as the tensor product of the underlying $R$-modules equipped with the multiplication naturally induced by those of $P$ and

$$
\begin{aligned}
Q:\left(x_{1} \otimes y_{1}\right) & \cdot\left(x_{2} \otimes y_{2}\right) \\
= & (-1)^{\left|y_{1}\right|\left|x_{2}\right|}\left(x_{1} \cdot x_{2}\right) \otimes\left(y_{1} \cdot y_{2}\right) .
\end{aligned}
$$

Definition 3.3. A graded Lie R-algebra ( or Lie $R$ superalgebra ) $\mathfrak{B}$ is a graded R-algebra, whose multiplication, called graded Lie bracket and denoted by [,], satisfies the following identities:

$$
\begin{aligned}
& {[x, y]=-(-1)^{|x||y|}[y, x]} \\
& (-1)^{|x||z|}[x,[y, z]]+(-1)^{|y||x|}[y,[z, x]]+ \\
& (-1)^{|z||y|}[z,[x, y]]=0 .
\end{aligned}
$$

Remark 3.4. Given a graded Lie algebra $\mathfrak{B}$, its even part $\mathfrak{B}_{0}$ is a Lie algebra over the ring $R_{0}$.

An important class of graded Lie algebras can be constructed in terms of the notion of graded derivation.

Let Pbe a graded-commutative R-algebra.

Definition 3.5. A homogeneous morphism $D \in \operatorname{End}_{R} P$ is a graded derivation of $P$ over $R$ if it fulfills the following condition (called the graded Leibnitz rule)

$D(x \cdot y)=D(x) \cdot y+(-1)^{|x||D|} x \cdot D(y)$.

The graded $R$-submodule of $\operatorname{End}_{R} P$ generated by the graded by the derivations of $P$ will be denoted by $\operatorname{Der}_{R} P$, or simply $\operatorname{Der} P$.

Proposition 3.6. DerP, equipped with the graded Lie bracket

$\left[D_{1}, D_{2}\right] \equiv D_{1} \circ D_{2}-(-1)^{\left|D_{1}\right|\left|D_{2}\right|} D_{2} \cdot D_{1}$,

is a graded Lie R-algebra.

By identifying $R$ with the submodule $R .1 \subset P$, condition (3.4) implies that, for all $D \in \operatorname{Der} P, D(R)=0$. We notice that $\operatorname{Der} P$ is a (left) graded $P$-module in a natural way, by letting $(x D)(y)=x \cdot D(y)$.

Definition 3.7. A graded derivation of $P$ over $R$ with values in $M$ is a homogeneous element $D \in \operatorname{Hom}_{R}(P, M)$ which fulfills a graded Leibnitz rule formally identical with equation (3.3).
The graded $P$-submodule of $\operatorname{Hom}_{R}(P, M)$ generated by the graded derivations of $P$ with values in $M$ will be denoted by $\operatorname{Der}_{R}(P, M)$.

Proposition 3.8. Let $M$ and $N$ be $R$-modules. There is a natural morphism of graded $R$-modules

$$
\phi: N \otimes M^{*} \rightarrow \operatorname{Hom}(M, N)
$$

described by $\phi(n \otimes \omega)(m)=n \omega(m)$. This induces $a$ morphism

$$
\gamma: M^{*} \otimes N^{*} \rightarrow(M \otimes N)^{*}
$$

whose expression is

$\gamma(\omega \otimes \eta)(m \otimes n)=(-1)^{|\eta||m|} \omega(m) \eta(n)$.

Both morphisms are bijective whenever $M$ is free and finitely generated.

Graded Exterior Algebra : Let $M$ be a graded $R$-module and let us denote by

$$
T^{p} M=M \underbrace{\otimes \cdots \otimes}_{P} M
$$

(3.1)

The $p$-th tensor power of $M$, graded as usual. We can consider as in the non-graded setting the graded tensor algebra of $M$,

$\mathcal{T}(M)=\underbrace{\infty}_{p=0} T^{p} M$,

which is in a natural way a bigraded $R$-algebra (i.e. it has the usual $\mathbb{Z}$-gradation of the tensor algebra, together with the $\mathbb{Z}_{2}$-gradation it carries as a graded $R$-algebra).

The graded exterior algebra $\wedge_{R} M$ of $M$ (denoted simply by $\wedge M)$ is defined as the quotient of $\mathcal{T}(M)$ by the graded ideal $\mathfrak{I}(M)$ generated by elements of the form $m_{1} \otimes m_{2}+$ $(-1)^{\left|m_{1}\right|\left|m_{2}\right|} m_{2} \otimes m_{1}$, with $m_{1}, m_{2}$ homogeneous. The product induced in $\wedge M$ by this quotient is denoted by $\wedge$ and is called the (graded) wedge product, as usual. If we let $\mathfrak{J}^{p}(M)=\mathfrak{I}(M) \cap T^{p} M$, since $\mathfrak{I}(M)$ is generated by homogeneous elements, we obtain $\mathfrak{I}(M)=\bigotimes_{p=0}^{\infty} \mathfrak{I}^{p}(M)$ and therefore,

$$
\wedge M=\bigotimes_{p=0}^{\infty} \wedge^{p} M
$$

with $\wedge^{p} M=T^{p} M / \mathfrak{I}^{p}(M)$.

We wish to ascertain the relationship existing between the exterior algebra $\wedge M^{*}$ and the modules of alternating graded multilinear forms: this will be realized by a morphism analogous to the morphism

$\gamma: M_{1}^{*} \otimes \cdots \otimes M_{n}^{*} \rightarrow\left(M_{1} \otimes \cdots \otimes M_{n}\right)^{*} \simeq$ $\mathcal{L}\left(M_{1}, \ldots, M_{n} ; R\right)$.

If $F_{p} \in \operatorname{HOm}\left(T^{p} M, R\right)$ and $F_{q} \in \operatorname{Hom}\left(T^{p} M, R\right)$ are homogeneous graded multilinear forms, $F_{p} \otimes F_{q}$ acts on a family of homogeneous elements according to the formula: 


$$
\begin{aligned}
& \left(F_{p} \otimes F_{q}\right)\left(m_{1}, \ldots, m_{p+q}\right) \\
& =(-1)^{\left|F_{q}\right|\left(\left|m_{p+1}\right|+\cdots+\left|m_{p+q}\right|\right)} F_{p}\left(m_{1}, \ldots, m_{n}\right) \\
& F_{q}\left(m_{p+1}, \ldots, m_{p+q}\right) .
\end{aligned}
$$

Let $\mathcal{S}_{p}$ be the group of permutation of $p$ objects. For any $\sigma \in \mathcal{S}_{p}$ and any $F_{p} \in \operatorname{Hom}\left(T^{p} M, R\right)$, we write, for homogeneous elements $m_{1}, \ldots, m_{p} \in M$,

$$
\begin{aligned}
& F_{p}^{\sigma}\left(m_{1}, \ldots, m_{p}\right) \\
& =(-1)^{\Delta_{1}(\sigma, m)} F_{p}\left(m_{\sigma(1)}, \ldots, m_{\sigma(p)}\right),
\end{aligned}
$$

where

$$
\Delta_{1}(\sigma, m)=\sum_{1 \leq i<j \leq p} \sum_{\sigma(i)>\sigma(j)}\left|m_{\sigma(i)}\right|\left|m_{\sigma(j)}\right| .
$$

Definition 3.9. A graded multilinerar form $F_{p} \in$ $\operatorname{Hom}\left(T^{p} M, R\right)$ is said to be alternating if $F_{p}^{\sigma}=(-1)^{|\sigma|} F_{p}$ for every $\sigma \in \mathcal{S}_{p}$, where $|\sigma|$ is the parity of the permutation $\sigma$.

The set $\operatorname{Alt}(M \underbrace{\times \cdots \times}_{p} M ; R) \equiv \operatorname{Alt}\left(M^{p}, R\right) \quad$ of all alternating graded multilinear forms is a submoduleof $\operatorname{Hom}\left(T^{p} M, R\right)$; we can introduce a projection morphism, which is no more than the graded anti-symmetrization:

$$
\begin{aligned}
& A_{p}: \operatorname{Hom}\left(T^{p} M, R\right) \rightarrow \operatorname{Alt}\left(M^{p} ; R\right) \\
& F_{p} \rightarrow A_{p}\left(F_{p}\right)=\frac{1}{p !} \sum_{\sigma \in \mathcal{S}_{p}}(-1)^{|\sigma|} F_{p}^{\sigma} .
\end{aligned}
$$

Proposition 2.10. The morphism $A_{p}$ has the following properties:

(a) $A_{p}\left(F_{p}\right)=F_{p}$ for any alternating form $F_{p}$;

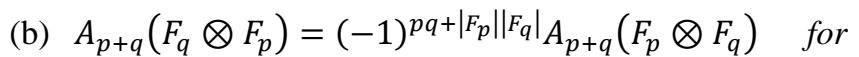
homogeneous $F_{p}, F_{q}$;

(c) $A_{p+q}\left(A_{p}\left(F_{p}\right) \otimes F_{q}\right)=A_{p+q}\left(F_{p} \otimes F_{q}\right)$.

We assume that $M$ is a free and finitely generated module, so that we may identify $T^{p}\left(M^{*}\right)$ with $\operatorname{Hom}\left(T^{p} M, R\right)$. In this way, the morphism $A_{p}$ yields the exact sequence of graded $R$-modules

$0 \rightarrow \mathfrak{I}\left(M^{*}\right) \rightarrow T^{p} M^{*} \stackrel{A_{p}}{\rightarrow} \operatorname{Alt}\left(M^{p} ; R\right) \rightarrow 0$,

and therefore we obtain an isomorphism $\wedge^{p} M^{*} \simeq$ $\operatorname{Alt}\left(M^{p} ; R\right)$. Thus, for a free and finitely generated module $M$, the homogeneous elements in the graded exterior algebra $\wedge M^{*}$ can be interpreted as alternating graded multilinear forms on $M$. In particular, we may interpret the wedge product of two elements $w^{p} \in \wedge^{p} M^{*}$ and $w^{q} \in$ $\wedge^{q} M^{*}$ as a graded multilinear form, which acts on homogeneous elements $m_{1}, \ldots, m_{p+q}$ according to [9];

$$
\begin{gathered}
\left(\omega^{p} \wedge \omega^{q}\right)\left(m_{1}, \ldots, m_{p+q}\right)=\frac{1}{(p+q) !} \\
\sum_{\sigma \in \mathcal{S}_{p}}(-1)^{|\sigma|+\Delta_{2}\left(\sigma, m, \omega^{q}\right)} \omega^{p}\left(m_{\sigma(1)}, \ldots, m_{\sigma(p)}\right) \\
\omega^{q}\left(m_{\sigma(p+1)}, \ldots, m_{\sigma(p+q)}\right)
\end{gathered}
$$

where in terms of the symbol $\Delta_{1}(\sigma, m)$ previously defined, we get

$\Delta_{2}\left(\sigma, m, \omega^{q}\right)=\Delta_{1}(\sigma, m)+\left|\omega^{q}\right| \sum_{i=1}^{p}\left|m_{\sigma(i)}\right|$.

\section{Matrices}

Given a graded-commutative ring $R$, an $R$-module morphism $R^{m \mid n} \rightarrow R^{p \mid q}$ can be regarded, relative to the canonical bases of $R^{m \mid n}$ and $R^{p \mid q}$, as a $(p+q) \times(m+n)$ matrix with entries in $R$,

$X=\left(\begin{array}{ll}X_{1} & X_{2} \\ X_{3} & X_{4}\end{array}\right)$

which acts on column vectors in $R^{n t \mid n}$ from the left. The set $M_{R}[(p+q) \times(m+n)]$ of such matrices can be graded so as to be naturally isomorphic to the graded $R$-module $\operatorname{Hom}_{R}\left(R^{m \mid n}, R^{p \mid q}\right)$, by decreeing that:

- $X$ is even if $X_{1}$ and $X_{4}$ have even entries, while $X_{2}$ and $X_{3}$ have odd entries;

- $X$ is odd if $X_{1}$ and $X_{4}$ have odd entries, while $X_{2}$ and $X_{3}$ have even entries

The set of matrices of the form (4.1), equipped with this gradation, will be denoted by $M_{R}[p|q ; m| n]$. The set of square matrices $M_{R}[m \mid n]$ (which are obtained by letting $p=m, q=n)$ is a graded $R$-algebra.

The usual notation of trace and determinant of a matrix can be expended to the matrices in $M_{R}[m \mid n]$, thus obtaining the concepts of graded trace and Berezinian (also called supertrace and superdeterminant respectively). For any matrix $\quad X \in M_{R}[p|q ; m| n], \quad$ regarded as a morphosm $X: R^{m \mid n} \rightarrow R^{m \mid n}$, we define the graded transpose of $X$-denoted by $X^{g t}$-as the matrix corresponding to the morphism $X^{*}:\left(R^{p \mid q}\right)^{*} \rightarrow\left(R^{m \mid n}\right)^{*}$ dual to $X$. With reference to equation (4.1), one obtains the following relations, where the superscript $t$ denotes the usual matrix transportation:

$\left(\begin{array}{ll}X_{1} & X_{2} \\ X_{3} & X_{4}\end{array}\right)^{g t}=\left\{\begin{array}{l}\left(\begin{array}{cc}X_{1}^{t} & X_{2}^{t} \\ -X_{3}^{t} & X_{4}^{t}\end{array}\right) \text { if }|X|=0 \\ \left(\begin{array}{cc}X_{1}^{t} & -X_{3}^{t} \\ X_{2}^{t} & X_{4}^{t}\end{array}\right) \text { if }|X|=1\end{array}\right.$

The graded transportation behaves naturally with respect to matrix multiplication:

$$
(X Y)^{g t}=(-1)^{|X||Y|} Y^{g t} X^{g t} .
$$


The graded trace of $X$ is the element $\operatorname{Str} X=\sum_{i} a_{i}^{*}\left(a^{i}\right) \in R$. Alternatively, one can give a direct characterization by letting, for all homogeneous $X \in M_{R}[m \mid n]$,

$S t r=\operatorname{Tr} X_{1}-(-1)^{|X|} \operatorname{Tr} X_{4}$

where $\operatorname{Tr}$ e designates the usual trace operation. The graded trace determines an $R$-module morphism Str: $M_{R}[m \mid n] \rightarrow$ $R$, which is natural with respect to graded transportation and matrix multiplication:

$$
\operatorname{Str}\left(X^{g t}\right)=\operatorname{Str} X
$$

$\operatorname{Str}(X Y)=(-1)^{|X||Y|} \operatorname{Str}(Y X)$.

Let us notice that, by denoting by $I_{m \mid n}$ the identity matrix, one has $\operatorname{Str} I_{m \mid n}=m-n$.

In order to extend the notion of determinant, we must consider the subgroup $G L_{R}[m \mid n]$ of the matrices in $M_{R}[m \mid n]$ corresponding to an even invertible endomorphisms. $G L_{R}[m \mid n]$ is the natural extension of the notion of general linear group, so that it will be called the general graded linear group.

Proposition 4.1. A matrix $x \in M_{R}[m \mid n]_{0}$ is in $G L_{R}[m \mid n]$ if and only if $X_{1} \in G L_{R}[m \mid 0]$ and $X_{4} \in G L_{R}[0 \mid n]$, i.e. $X$ is invertible if and only if $X_{1}$ and $X_{4}$ are invertible as ordinary matrices with entries in $R_{0}$.

Definition 4.2. [1], [3], [4] Let $X \in G L_{R}[m \mid n]$. the Berezinian of $X$ is the element in $G L_{R}[1 \mid 0]$ given by

$$
\operatorname{Ber} X=\left(\begin{array}{ll}
X_{1} & X_{2} \\
X_{3} & X_{4}
\end{array}\right)
$$

$=\operatorname{det}\left(X_{1}-X_{2} X_{4}^{-1} X_{3}\right)\left(\operatorname{det} X^{-1}\right)$.

Proposition 4.3. The mapping Ber: $G L_{R}[m \mid 0] \rightarrow$ $G L_{R}[0 \mid n]$ is a group morphism, that coincides with the determinant whenever $n=0$ :

$\operatorname{Ber}(X Y)=\operatorname{Ber} X \operatorname{Ber} Y \quad \forall X, Y \in G L_{R}[m \mid n]$

Theorem 4.4. A matrix in $X \in M_{R}[m \mid n]_{0}$ is invertible if and only if $\sigma(X) \in G L[m+n]$.

Proof. The 'only if' part is trivial, since $\sigma$ is ring morphism. To show the converse, it suffices to prove that a matrix $Z \in M_{B_{L}}[p \mid 0]_{0}$ is invertible as a matrix with entries in $\left(B_{L}\right)_{0}$ if $\sigma(Z)$ is invertible. In the case $p=1$ this is a consequence of the fact that in $B_{L}$ the morphism $\sigma$ is the natural projection $\left(B_{L}\right)_{0} \rightarrow\left(B_{L}\right)_{0} /\left(n_{L}\right)_{0}$. The result is easily extended to $p>1$ by inclusion.

\section{Conclusion}

We start with given an arbitrary group $G$ and introducing $G$ graded algebraic objects and for a given gradedcommutative ring $R$ and $R$-module morphism can be regarded, relative to the canonical bases of relative to the canonical bases of $R^{m \mid n}$ and $R^{p \mid q}$, as a $(p+q) \times(m+n)$ matrix with entries in $R, X=\left(\begin{array}{ll}X_{1} & X_{2} \\ X_{3} & X_{4}\end{array}\right)$, which acts on column vectors in $R^{n t \mid n}$ from the left. Finally, this article induces a Theorem 4.4.on a matrix of graded $R$-algebra. This paper will be helpful for other researchers.

\section{References}

1. Arnowitt, R., Nath, P., Zumino, B. 1975. Superfield densities and action principle in superspace, Phys. Lett. 56B, 81-84.

2. Atiyah, M. F., Macdonal, I. G. 1969. Introduction to commutative algebra, Addision-Wesley, Reading, MA.

3. Bartocci, C., Bruzzo, U., Hernández Ruipérez, D. 1991. The geometry of supermanifolds. Kluwer, Dordrecht,.

4. Berezin, F. A., Leĭtes, D.A. 1975. Supermanifolds, Soviet Math. Dokl. 16,1218-1222.

5. Bourbaki, N. 1970. Elément de mathématique. AlgèbreI(Chapitres 1 à 3), Hermann, Parish.

6. Corwin, L., Ne'eman, Y., Sternberg, S. 1975. Graded Lie algebras in mathematics and physics (Bose-Fermi symmetry), Rev. Modern Phys. 47, 573-603.

7. Kac, V.G. 1977. Lie superalgebras, Adv. In Math. 26, 8-96.

8. Kac, V.G. 1977, A sketch of Lie Superalgebra theory, Commun. Math. Phys.53, 31-64.

9. Kobayashi, S., Nomizu, K. 1963. Foundations of differential geometry. I, Inter-science Publ., New York.

10. Năstăsescu, C., Van Oystaeyen, F. 1982. Graded ring theory, North-Holland, Amsterdam.

11. Scheunert, M. 1979. The theory of Lie Superalgebra, Lecture Notes Math., Springer Verlag, Berlin,716. 
